\title{
Impacto de la pandemia en el manejo de la hernia inguinal en lactantes
}

\author{
C. de la Sen Maldonado, N. Albertos Mira-Marcellí, V. Díaz Díaz, M.G. Toro Rodríguez, J. Gonzálvez Piñera \\ Servicio de Cirugía Pediátrica. Hospital General Universitario de Alicante.
}

\begin{abstract}
RESUMEN
Introducción. La pandemia del SARS-CoV-2 supuso una reducción de las jornadas quirúrgicas. Analizamos el efecto en la morbilidad de la hernia inguinal.

Material y método. Estudio retrospectivo de casos y controles. Incluimos menores de 18 meses intervenidos de hernia inguinal desde 01/01/2019 hasta 31/08/2020, divididos en dos grupos: intervenidos antes (grupo A) o después (grupo D) de la declaración del estado de alarma. Variables principales: episodios de incarceraciones y complicaciones postoperatorias. Variable secundaria: intervención urgente o programada. Variables poblacionales: sexo, edad gestacional (EG), patología previa, edad al diagnóstico (ED) y a la intervención (EI) (meses).
\end{abstract}

Resultados. Incluimos 64 pacientes, 43 grupo A y 21 grupo D. En el grupo A la mediana de EG fue $37+3$, el 90,5\% fueron varones, la mediana ED fue 2,33 y EI 3,27. En el grupo B la mediana de EG fue $31+2$, el 72,1\% fueron varones, la mediana ED fue 3 y EI 3,63. No hubo diferencias estadísticamente significativas. Sí fueron significativas las diferencias en patología previa: $16,3 \%$ A y $38,1 \% \mathrm{D}(\mathrm{p}=0,05)$. Sobre la variable principal, 25,6\%, del A sufrieron incarceraciones frente a $33,3 \%$ del $\mathrm{D}(\mathrm{p}=0,51)$ y un $9,3 \%$ tuvieron morbilidad postoperatoria en A frente al 14,3\% en D ( $p=0,41)$. Respecto a la variable secundaria, un $88,4 \%$ se realizaron de forma programada en el grupo A y un $90,5 \%$ en el grupo $\mathrm{D}(\mathrm{p}=0,583)$.

Conclusión. Pese a la reducción de jornadas quirúrgicas, no ha aumentado la morbilidad de la hernia inguinal en nuestro medio. Hemos observado un aumento de pacientes con patología previa, implicando la priorización de los pacientes más graves.

Palabras Clave: Hernia inguinal infantil; Cirugía pediátrica; SARSCoV-2.

\section{PANDEMIC'S IMPACT ON INGUINAL HERNIA MANAGEMENT IN INFANTS}

\begin{abstract}
Introduction. The SARS-CoV-2 pandemic brought about a reduction in surgical activity. The objective of this work was to analyze its impact on inguinal hernia morbidity.

Materials and methods. A retrospective study of cases and controls was carried out. Patients under 18 months of age undergoing inguinal hernia surgery from January 1, 2019 to August 31, 2020 were included. They were divided into two groups: patients undergoing surgery before (group A) or after (group D) the state of alarm was declared in Spain. Primary variables: episodes of incarceration and postoperative complications. Secondary variable: urgent or scheduled surgery. Demographic variables: sex, gestational age (GA), previous pathologies, age at diagnosis (AD), and age at surgery (AS) (months).

Results. 64 patients were included -43 in group A and 21 in group D. In group A, median GA was $37+3,90.5 \%$ of patients were male, median $\mathrm{AD}$ was 2.33 , and median $\mathrm{AS}$ was 3.27. In group $\mathrm{D}$, median $\mathrm{GA}$ was $31+2,72.1 \%$ of patients were male, median $\mathrm{AD}$ was 3 , and median AS was 3.63. There were no statistically significant differences. However, differences in terms of previous pathologies were significant (16.3\% for Group A vs. $38.1 \%$ for group D) $(\mathrm{p}=0.05)$. Regarding the primary variable, $25.6 \%$ of patients in group A had incarcerations $v s .33 .3 \%$ of patients in group $\mathrm{D}(\mathrm{p}=0.51)$, whereas $9.3 \%$ of patients in group A had postoperative morbidity $v s .14 .3 \%$ of patients in group $\mathrm{D}(\mathrm{p}=0.41)$. Regarding the secondary variable, $88.4 \%$ of surgeries in group A were scheduled vs. $90.5 \%$ of surgeries in group D $(\mathrm{p}=0.583)$.

Conclusion. In spite of reduced surgical activity, inguinal hernia morbidity did not surge in our environment. An increase in patients with previous pathologies was noted, which means severe patients should be prioritized.
\end{abstract}

KeY Words: Inguinal childhood hernia; General surgery; Pediatrics; SARS-CoV-2.

\section{INTRODUCCIÓN}

La hernia inguinal es una de las patologías más habituales en cirugía pediátrica, con una prevalencia aproximada del $5 \%$ en niños varones. Su incidencia es máxima en niños menores de un año y el riesgo de incarceración también es mayor en 
Tabla I. Variables demográficas cuantitativas y significación estadística.

\begin{tabular}{|c|c|c|c|c|c|c|c|}
\hline & \multicolumn{3}{|c|}{ Grupo A } & \multicolumn{3}{|c|}{ Grupo D } & \multirow[b]{2}{*}{$p$} \\
\hline & Mediana & Intervalo d & ianza $95 \%$ & Mediana & Intervalo o & ianza $95 \%$ & \\
\hline SEG & 37,30 & 36,00 & 38,00 & 31,10 & 29,40 & 37,00 & 0,073 \\
\hline Edad al diagnóstico en meses & 2,33 & 2,16 & 2,96 & 2,96 & 1,40 & 3,96 & 0,902 \\
\hline Edad a la intervención en meses & 3,28 & 2,80 & 4,23 & 3,58 & 2,60 & 4,83 & 0,374 \\
\hline
\end{tabular}

esta franja de edad ${ }^{(1)}$. Los factores de riesgo de complicación de las hernias inguinales son bien conocidos, destacando que el tiempo de espera desde el diagnóstico hasta la intervención es un factor de riesgo independiente de incarceración ${ }^{(2)}$, con el aumento de morbilidad que esto conlleva.

La pandemia del SARS-CoV-2 ha supuesto un desafío sin precedentes para nuestro sistema sanitario. No solo para asistir a la población afecta por este, sino también para atender a los pacientes con otras patologías en un contexto sociosanitario tan peculiar, con las restricciones que impuso el estado de alarma. La asistencia sanitaria se vio modificada forzosamente hasta reducir las jornadas quirúrgicas programadas, así como minimizar la presencialidad de las consultas médicas. Una de las preocupaciones de los profesionales que hemos atendido población pediátrica en esta etapa es el impacto que este posible retraso de diagnóstico y de tratamiento haya podido tener sobre nuestros pacientes.

Por todo ello, el objetivo de nuestro trabajo es evaluar si las reorganizaciones asistenciales que ha impuesto la pandemia del SARS-CoV-2 han repercutido en un incremento de la morbilidad en los lactantes intervenidos de hernia inguinal en nuestro medio.

\section{MATERIAL Y MÉTODOS}

Se realizó un estudio observacional y retrospectivo de casos y controles revisando las historias clínicas de todos los pacientes menores de 18 meses a los que se realizó una herniorrafia inguinal en el Servicio de Cirugía Pediátrica desde el 01/01/2019 hasta el 31/08/2020, dividiéndolos en dos grupos según si la fecha de su intervención fue antes (grupo A) o después (grupo D) de la declaración del estado de alarma (14 de marzo de 2020)(3), fecha que coincidió con la suspensión de toda actividad quirúrgica programada en nuestro medio.

Se excluyeron aquellos pacientes en los que la historia clínica fuese incompleta y no permitiese la obtención de todos los datos clínicos del estudio.

Como variables demográficas se recogieron: sexo, edad gestacional en semanas (EG), patología previa (patología cardiaca, respiratoria o neurológica mayor), edad al diagnóstico en meses (ED) y edad a la intervención en meses (EI).

Las variables resultado principales del estudio fueron: la morbilidad preoperatoria (contabilizada como episodios de incarceración antes de la intervención) y la morbilidad postoperatoria (es decir, las complicaciones postoperatorias), ambas como variables cualitativas. Como variable secundaria se analizó si la intervención había sido realizada de forma urgente o programada.

La recogida de datos se realizó mediante la revisión de las historias clínicas electrónicas, solicitándose al servicio de documentación clínica todos los pacientes con diagnóstico operatorio de hernia inguinal (código K40 en CIE-10) o procedimiento herniorrafia inguinal (códigos 0YQ50ZZ, 0YQ60ZZ y 0YQA0ZZ) durante este periodo de tiempo y la cumplimentación de un formulario realizado mediante el programa Microsoft Office Access ${ }^{\odot}$.

El estudio de contraste de hipótesis se realizó mediante el programa IBM SPSS Statistics v25 ${ }^{\circ}$. Los test estadísticos utilizados fueron: test de chi-cuadrado o test exacto de Fisher para las variables cualitativas y la t de Student o la U de Mann-Whitney para las variables cuantitativas. Como medida de riesgo se calculó la odss ratio con un intervalo de confianza al $95 \%$. Se consideraron estadísticamente significativas las diferencias si su significación fue menor del 0,05 ( $\mathrm{p}<0,05)$.

\section{RESULTADOS}

Se encontraron 68 pacientes que cumplían criterios de inclusión, de los cuales 4 fueron excluidos debido a que la historia clínica era incompleta. De estos, 43 pacientes se intervinieron antes de la declaración del estado de alarma, constituyendo el grupo A, y 21 se intervinieron después de la declaración del estado de alarma, constituyendo el grupo D.

En el grupo A, el 90,5\% fueron varones, un 16,3\% presentaban patología previa, la mediana de edad gestacional fue de 37,3 semanas, la mediana de edad al diagnóstico fue de 2,3 meses y la mediana de edad a la intervención fue de 3,3 meses.

En el grupo D, el 72,1\% fueron varones, el 38,09\% presentaban patología previa, la mediana de edad gestacional fue de 31,2 semanas, la mediana de edad al diagnóstico fue de 2,9 meses y la mediana de edad a la intervención fue de 3,58 meses.

En la tabla I se muestran las características demográficas de ambos grupos. Las diferencias entre ellas no fueron estadísticamente significativas, con la excepción de la proporción de pacientes con patología previa concomitante $(\mathrm{p}=0,035)$. 
Tabla II. Variable resultado principal morbilidad

preoperatoria, significación y medida de riesgo.

\begin{tabular}{lcc} 
& Grupo A & Grupo D \\
\hline Incarceración sí & 11 & 7 \\
\hline Incarceración no & 32 & 14 \\
\hline $\mathrm{p}=0,51$ & OR $=1,61$ (IC95\% & $0,514-5,07)$
\end{tabular}

Respecto a las variables resultado principales:

- Un 25,6\% de pacientes del grupo A sufrieron al menos un episodio de incarceración frente al 33,3\% en el grupo D; odds ratio (OR) de 1,61 (intervalo de confianza (IC) al 95\% 0,514-5,07), no alcanzando significación estadística $(\mathrm{p}=0,51)$.

- Un 9,3\% de los pacientes del grupo A presentaron complicaciones postoperatorias frente al 14,3\% en el grupo D, OR 1,625 (IC95\% 0,329-8,030), no alcanzando significación estadística $(\mathrm{p}=0,41)$.

En las tablas II y III se muestran los resultados de estas variables.

Respecto a la variable secundaria: un 11,6\% de las intervenciones se realizaron de forma urgente en el grupo $\mathrm{A}$ y un $9,5 \%$ en el grupo $\mathrm{D}$, sin alcanzar significación estadística $(\mathrm{p}=0,583)$.

\section{DISCUSIÓN Y CONCLUSIONES}

Actualmente empiezan a surgir numerosos estudios que analizan el impacto que la pandemia del SARS-CoV-2 ha supuesto en la salud de la población, tanto por su alta tasa de mortalidad en población frágil como el aumento de la morbimortalidad de la patología grave no-COVID debido a un retraso en la atención sanitaria ${ }^{(4,5)}$.

A nivel quirúrgico, muchos estudios han evidenciado una reducción en el número de procedimientos urgentes realizados durante la pandemia ${ }^{(6-9)}$, junto a este hecho también se ha observado en algunos estudios un aumento del tiempo de evolución hasta recibir tratamiento debido al retraso diagnóstico y un aumento de la morbimortalidad(9).

También destaca un estudio de mayo de 2020 que estimó que "durante el pico de 12 semanas del COVID-19 se suspendieron o pospusieron 28.404 .603 intervenciones quirúrgicas, lo cual precisaría de una media de 45 semanas a un $120 \%$ del volumen de trabajo habitual de recuperar" ${ }^{\prime 10}$, mostrando que los efectos que la pandemia del SARS-CoV-2 ha tenido sobre la atención sanitaria tardarán en resolverse.

Múltiples artículos evidencian que el riesgo de incarceración aumenta con el retraso diagnóstico ${ }^{(1,11-14)}$; así, Zamakhshary $\mathrm{M}$ y cols. observaron que los pacientes que tardaron más de 14 días desde el diagnóstico hasta la intervención presentaron el doble de riesgo de padecer un episodio de incarceración $^{(2)}$.
Tabla III. Variable resultado principal morbilidad postoperatoria, significación y medida de riesgo.

\begin{tabular}{lcc} 
& Grupo A & Grupo D \\
\hline Morbilidad postoperatoria sí & 4 & 3 \\
\hline Morbilidad postoperatoria no & 39 & 118 \\
\hline $\mathrm{p}=0,41$ & $\mathrm{OR}=1,625$ (IC95\% 0,329-8,030)
\end{tabular}

En el momento de la redacción de este artículo, revisando la literatura no se encontraron artículos de cirugía pediátrica que estudiasen la morbilidad de la hernia inguinal en niños en época de pandemia.

Sí se encontraron tres artículos sobre pacientes adultos. Destacar el artículo de Lima DL y cols. ${ }^{(15)}$, en el cual observaron una reducción estadísticamente significativa del número de intervenciones urgentes, así como el número de consultas relacionadas con patología herniaria durante la época de pandemia.

En nuestra muestra hemos observado que los pacientes que fueron intervenidos durante la pandemia presentaban un mayor porcentaje de patología previa y una menor edad gestacional. Consideramos que esto es debido a que, dada la limitación de jornadas quirúrgicas, hemos priorizado a los pacientes más complejos para reducir en la medida de lo posible el riesgo de complicaciones en estos pacientes.

Otra posible explicación es que los pacientes más complejos han llegado en número similar, al ser derivados normalmente desde unidades de neonatología que no han visto tan restringida su actividad. Sin embargo, los pacientes más leves que normalmente provienen de Atención Primaria habrían llegado en menor número por las mayores restricciones sufridas en la pandemia en los centros de salud.

También se aprecia un leve aumento en la edad al diagnóstico y a la intervención. Esto concuerda con que los pacientes hayan sido diagnosticados más tarde y, por ende, se hayan intervenido con mayor edad.

Observamos también un aumento en la morbilidad perioperatoria, especialmente con un aumento de los episodios de incarceración. Esto concuerda con que el retraso en el diagnóstico y tratamiento aumenta el riesgo de incarceración y de complicaciones postoperatorias en la hernia inguinal ${ }^{(1,2)}$. Debemos destacar que las diferencias observadas no han sido significativas en el estudio de contraste de hipótesis. Esto podría deberse a que, dado el limitado tamaño muestral de nuestro estudio, las diferencias no son lo suficientemente grandes como para ser detectadas, o que no existen diferencias reales.

No observamos aumento de la proporción de intervenciones realizadas de forma urgente. En nuestro estudio, la única indicación para realización de cirugía urgente fue la imposibilidad de reducir una incarceración mediante maniobras de taxis. Dado que no ha aumentado la proporción de hernias 
intervenidas de forma urgente, asumimos que el éxito en la reducción de las mismas se ha mantenido estable.

En cuanto a posibles sesgos y limitaciones de nuestro estudio, primeramente, debemos destacar que el tamaño muestral es pequeño, por lo que son necesarias diferencias clínicas muy grandes para alcanzar significación estadística. Sería necesario realizar estudios con mayor número de pacientes, especialmente multicéntricos, para poder extraer conclusiones con mayor evidencia.

También al ser un estudio retrospectivo existen otros posibles puntos de sesgo, como la pérdida de pacientes por no estar bien codificados en la historia clínica electrónica. $\mathrm{O}$, a pesar de la codificación correcta, que el registro sea incompleto, no pudiendo extraer la información de las variables.

Otro punto a tener en cuenta es que el grupo D presenta un tiempo de seguimiento más corto, por lo que pueden no observarse los efectos a largo plazo.

Como conclusión destacamos que, pese a la reducción de jornadas quirúrgicas y de visitas a consultas externas que ha supuesto la pandemia, no hemos observado un aumento en la morbilidad pre- ni postoperatoria de los pacientes intervenidos de hernia inguinal. Además, los pacientes en este periodo de tiempo presentaban un mayor porcentaje de patología previa y menor edad gestacional, siendo pacientes con mayor riesgo de complicaciones. Esto implica que hemos priorizado a los pacientes más complejos al vernos limitados en el número de pacientes que podíamos intervenir.

\section{BIBLIOGRAFÍA}

1. Zamakhshary M, To T, Guan J, Langer JC, et al. Risk of incarceration of inguinal hernia among infants and young children awaiting elective surgery. CMAJ. 2008; 179: 1001-5.

2. Sulkowski JP, Cooper JN, Duggan EM, et al. Does timing of neonatal inguinal hernia repair affect outcomes? J Pediatr Surg. 2015; 50: 171-6.

3. Real Decreto 463/2020, de 14 de marzo, por el que se declara el estado de alarma para la gestión de la situación de crisis sanitaria ocasionada por el COVID-19. Ref. BOE-A-2020-3692.
4. Blecker S, Jones SA, Petrilli CM, et al. Hospitalizations for chronic disease and acute conditions in the time of COVID-19. JAMA Intern Med. 2021; 181(2): 269-71.

5. Bhambhvani HP, Rodrigues AJ, Yu JS, Carr JB II, Hayden Gephart M. Hospital volumes of 5 medical emergencies in the COVID-19 pandemic in 2 US medical centers.JAMA Intern Med. 2021; 181(2): $272-4$.

6. Gupta R, Gupta J, Ammar H. Impact of COVID-19 on the outcomes of gastrointestinal surgery. Clin J Gastroenterol [Internet]. 2021; 14: 932-46. Available from: https://doi.org/10.1007/s12328-021-01424-4

7. Cano-Valderrama O, Morales X, Ferrigni CJ. Reduction in emergency surgery activity during COVID-19 pandemic in three Spanish hospitals. Br J Surg. 2020; 107(8): e239.

8. Patriti A, Eugeni E, Guerra F. What happened to surgical emergencies in the era of COVID-19 outbreak? Considerations of surgeons working in an Italian COVID-19 zone. Updates Surg. 2020; 72(2): 309-10.

9. Cano-Valderrama O, Morales X, Ferrigni CJ, et al. Acute care surgery during the COVID-19 pandemic in Spain: changes in volume, causes and complications. A multicentre retrospective cohort study. Int J Surg. 2020; 80: 157-61.

10. COVIDSurg Collaborative. Elective surgery cancellations due to the COVID-19 pandemic: global predictive modelling to inform surgical recovery plans. Br J Surg. 2020; 107(11): 1440-9.

11. Sulkowski JP, Cooper JN, Duggan EM, Balci O, Anandalwar SP, Blakely ML, et al. Does timing of neonatal inguinal hernia repair affect outcomes? J Pediatr Surg. 2015; 50(1): 171-6.

12. Wang KS, Papile LA, Baley JE, Benitz W, Cummings J, Carlo WA, et al. Assessment and management of inguinal hernia in infants. Pediatrics. 2012; 130(4): 768-73.

13. Espósito C, Escolino M, Turrà F, Roberti A, Cerulo M, Farina A, et al. Current concepts in the management of inguinal hernia and hydrocele in pediatric patients in laparoscopic era. Semin Pediatr Surg [Internet]. 2016; 25(4): 232-40. Available from: http://dx.doi. org/10.1053/j.sempedsurg.2016.05.006

14. Duggan EM, Patel VP, Blakely ML. Inguinal hernia repair in premature infants: More questions than answers. Arch Dis Child Fetal Neonatal Ed. 2015; 100(4): F286-8.

15. Lima DL, Pereira X, dos Santos DC, Camacho D, Malcher F. Where are the hernias? A paradoxical decrease in emergency hernia surgery during COVID-19 pandemic. Hernia. 2020; 24(5): 1141-2. 